\title{
Awareness and knowledge of HPV and cervical cancer in female students: a survey (with a
}

cautionary note)

Running title: Student knowledge of HPV and cervical cancer

Keywords: HPV awareness, cervical cancer, students, women, online survey

Susan M. Sherman ${ }^{1} \mathrm{PhD}$, Emma Nailer ${ }^{1} \mathrm{MSc}$, Clare Minshall ${ }^{1} \mathrm{BSc}$, Rachel Coombes ${ }^{1} \mathrm{BSc}$, Jason Cooper $^{2} \mathrm{MD}$, and Charles W. E. Redman ${ }^{2} \mathrm{MD}$

${ }^{1}$ School of Psychology, Keele University, Keele, Staffs, ST5 5BG, UK.

${ }^{2}$ University Hospital of North Staffordshire, Stoke on Trent, ST4 6QG, UK

We received no financial support for this study.

Corresponding author: Susan M. Sherman, School of Psychology, Dorothy Hodgkin Building, Keele University, Keele, Staffordshire, ST5 5BG, United Kingdom. Tel: 01782733384.

Electronic mail may be sent to s.m.sherman@keele.ac.uk. 


\section{SUMMARY}

We conducted a survey to explore levels of awareness and knowledge of HPV and cervical cancer in 170 female students and whether mode of data collection (online vs. paper) affected the results. $27 \%$ of women named HPV as a cause of cervical cancer with online respondents more likely to do so. $75 \%$ of women had heard of HPV. More online respondents had heard of HPV than paper respondents. 127 women reported having heard of HPV, with a mean knowledge score of 2.989 (SD = 1.599). Online respondents scored higher $(3.57, \mathrm{SD}=1.316)$ than paper respondents $(2.688, \mathrm{SD}=1.591)$. Knowledge and awareness of HPV and its link to cervical cancer appear to have increased which may be related to the HPV vaccination programme. However, there is still a considerable number of women with little to no knowledge of HPV. Online surveys may result in an inflated estimation of awareness and knowledge. 


\section{INTRODUCTION}

Human papillomavirus (HPV), a sexually transmitted infection, has been established as a cause in almost all cases of cervical cancer (WHO/ICO Information Centre on HPV and Cancer, 2013). Yet previous research has consistently shown low levels of awareness and knowledge of the virus and its link with cervical cancer. In face to face and paper-based surveys of UK individuals between $24 \%$ and $31 \%$ of adults reported being aware of HPV (Pitts \& Clarke, 2002; Phillips et al., 2003; Waller et al., 2003; Marlow et al., 2007) whilst between $0.6 \%$ and $2.5 \%$ of people named HPV as a risk factor or cause of cervical cancer in an open-ended question (Waller et al, 2004; Marlow et al., 2007). Even when people report being aware of HPV knowledge may still be poor. In one paper-based study, of the $30 \%$ of respondents who were aware of $\mathrm{HPV}$, only $16.8 \%$ displayed good knowledge on subsequent questions (Pitts \& Clarke, 2002).

Younger women particularly demonstrate lower levels of awareness and knowledge of HPV. Women aged 16-35 years are less likely to have heard of HPV than those aged over 36 years $^{5}$. Similarly, awareness of risk factors for cervical cancer is lowest in those aged 1624 years (or 75 years or over)(Waller et al., 2004).

In 2008, the HPV vaccination programme was introduced in the UK for girls aged 12 to 13 , with catch-up programmes offering the vaccine to all girls born on/after September $1^{\text {st }} 1990$. In 2011 the NHS cervical screening programme began to introduce HPV testing for women with borderline or mild dyskaryosis and, since 2013, HPV primary screening is being tested in some areas of England, with cytology only being carried out on samples following a positive HPV test. 
It is therefore feasible that awareness, knowledge and understanding of HPV and its role in cervical cancer may be improving. An online study comparing international rates of knowledge of HPV and HPV vaccination indicated greater awareness of HPV than previous studies, with $88 \%$ of US women, $72 \%$ of Australian women and $62 \%$ of UK women reporting awareness and most of those knowing that HPV causes cervical cancer (Marlow et al., 2013). However, research comparing online and offline survey responses to a knowledge-based question about cholesterol found that online respondents were better informed than the face to face respondents (Duffy et al, 2005), raising the possibility that online respondents might use the internet to look up answers potentially inflating the true extent of public understanding.

The primary purpose of this paper is to explore awareness and knowledge of HPV and cervical cancer in university female students in the UK. A secondary consideration is to compare data collected by paper and online questionnaires.

\section{METHODS}

\section{Participants}

Participants consisted of 170 females students aged between 18 and 22 years most of whom were recruited from Keele University. Participants responded to either an online questionnaire or a paper questionnaire. For the online questionnaire, participants were recruited through personal contacts of the 3rd and 4th authors and through social media and were provided with a link to the online survey. For the paper questionnaire, students were approached on campus and asked if they would like to take part in a survey. Half of the participants in each condition were asked not to collude or to look the answers up. 


\section{Materials and Procedure}

A 19 item self-report questionnaire was used to assess knowledge of cervical cancer and $H^{\prime} V^{i}$. This was available in two formats: a paper-based questionnaire and an online questionnaire, with both versions using identical questions presented in an identical order. The questionnaire consisted of three sections: section A collected socio-demographic information; section B asked about knowledge about the causes of cervical cancer; and section C referred to knowledge about HPV. The study received approval from the School of Psychology Ethics Committee at Keele University.

Section B consisted of two open-ended questions adapted from Marlow et al (2007) asking participants about their knowledge of the cause of cervical cancer. The first question asked specifically about what the participant considered to be the main cause of cervical cancer;' Thinking of cervical cancer, what do you think is its main cause?' The following question asked the participant to list as many other causes that they could think of; 'What other causes of cervical cancer, if any, are you aware of? Please write down as many as you can think of.'

The first question in section C asked participants if they had heard of HPV. If they responded 'yes' or 'don't know' they were instructed to continue answering all subsequent questions. These included closed questions asking what the letters HPV stand for, what HPV is, how HPV is contracted, what the relationship between HPV and cervical is and whether the HPV vaccine will prevent all cases of cervical cancer. These five questions were used to give participants a knowledge score out of 5 . There was also an open question, asking participants for any other knowledge they have of HPV and participants were also asked to indicate the sources of their knowledge about HPV. 
If participants indicated that they had not heard of HPV, they were instructed to bypass the HPV knowledge questions. The final two closed questions asked participants to indicate their personal involvement with HPV vaccinations, smear tests and cervical cancer.

\section{RESULTS}

Of the 170 participants, who were aged between 18 and 22 years $(M=20.17, S D=1.026)$, 84 responded to an online questionnaire $(M=20.07, S D=1.050)$ and 86 responded to a paper questionnaire $(M=20.27, S D=0.999)$. All participants were female university students and, as such, had similar levels of education and income. Only $11.2 \%$ of participants reported having a job alongside their studies and the majority of participants were single (93.5\%) and white (95.9\%). Chi-squared analyses showed no significant differences between the two groups for any of the demographics (see Table 1).

Differences in the causes of cervical cancer named, awareness of HPV, knowledge of HPV and sources of information about HPV for online and paper respondents were identified using chi-squared analyses. An overall knowledge score (out of 5) was calculated for each participant who had heard of HPV and mean scores for the two groups were compared using an independent t-test. For those who had heard of HPV, the number of blank or 'don't know' responses were also calculated for each HPV knowledge question. Chisquared analyses were used to identify differences for online and paper respondents and the mean number of blank or 'don't know' responses given by partcipants in each group was compared using an independent t-test.

\section{Causes of cervical cancer}


The two open questions about the causes of cervical cancer resulted in a range of responses summarised in Table 2. Just over a quarter of participants (27\%) named HPV as a cause of cervical cancer and $21 \%$ of women named an unspecified STI/STD as a cause. Compared to women who responded to the paper questionnaire, online respondents were more likely to name HPV as a cause $\left(\chi^{2}(1)=5.533, P=0.002 ; \mathrm{OR}=2.307,95 \% \mathrm{Cl}=1.140-4.669\right)$, more likely to name an unspecified STI/STD as a cause $\left(\chi^{2}(1)=4.686, P=0.030 ; \mathrm{OR}=2.325,95 \%\right.$ $\mathrm{Cl}=1.070-5.051)$, and were more likely to name either HPV or an STI/STD as a cause $\left(\chi^{2}(1)\right.$ $=9.517, P=0.002 ; \mathrm{OR}=2.663,95 \% \mathrm{Cl}=1.420-4.995)$. At least one sexual behaviour (e.g. unprotected sex, sexual activity, multiple sexual partners and young age of sexual activity) was named as a cause by $22 \%$ of women but there were no differences in mode of response for these causes.

At least one other biological factor was named as a cause of cervical cancer by $52 \%$ of women. The most common of these was genetics/family history which was more likely to be named as a cause by online respondents $\left(\chi^{2}(1)=5.182, P=0.023 ; \mathrm{OR}=2.170,95 \% \mathrm{Cl}=\right.$ $1.107-4.255)$, whilst an unspecified virus or infection was more likely to be named as a cause by paper respondents than by online respondents $\left(\chi^{2}(1)=8.567, P=0.003\right.$; OR $=$ $7.301,95 \% \mathrm{Cl}=1.594-33.442)$. Other biological factors, such as abnormal cells/mutations, poor health/weakened immune system and age, did not differ according to mode of response.

Just over a quarter of women (27\%) named at least one lifestyle factor as a cause of cervical cancer, with online respondents being more likely to do so $\left(\chi^{2}(1)=7.289, P=0.007\right.$; $\mathrm{OR}=2.630,95 \% \mathrm{Cl}=1.288-5.369)$. In particular, online respondents were more likely to name smoking as a cause $\left(\chi^{2}(1)=4.563, P=0.033 ; \mathrm{OR}=2.501,95 \% \mathrm{Cl}=1.059-5.904\right)$ and 
to name the contraceptive pill as a cause $\left(\chi^{2}(1)=6.979, P=0.008 ; \mathrm{OR}=6.329,95 \% \mathrm{Cl}=\right.$ 1.358 - 29.490). Other lifestyle factors named included lifestyle/diet/alcohol consumption, stress, number of chidlren, sun/sunbeds and poor hygiene, but these did not differ according to mode of response.

Approximately $12 \%$ of participants gave no response or answered 'don't know' to both of the questions about the causes of cervical cancer, with paper respondents being more likely to do so $\left(\chi^{2}(1)=6.283, P=0.012 ; \mathrm{OR}=3.611,95 \% \mathrm{Cl}=1.258-10.366\right)$.

\section{Awareness and knowledge of HPV}

In total, $75 \%$ of women reported having heard of HPV, with $85 \%$ of online respondents having heard of it and $65 \%$ of paper respondents having done so. Online respondents were more likely to have heard of HPV than paper respondents $\left(\chi^{2}(1)=8.470, P=0.004\right.$; OR $=$ $2.926,95 \% \mathrm{Cl}=1.397-6.127)$.

Responses to the questions regarding knowledge of HPV are summarised in Table 3. Of the 127 women who reported having heard of HPV, $50 \%$ scored at least 4 out of 5 and $68 \%$ scored at least 3 out of 5 on the HPV knowledge questions, with a mean score of 2.989 $(\mathrm{SD}=1.599)$. Mean scores differed according to mode of response $(\mathrm{t}(106)=3.347, P=0.001)$ with online respondents scoring a mean of $3.57(S D=1.316)$ compared to a mean of 2.688 $(S D=1.591)$ for paper respondents.

Answers to the question 'What do the letters HPV stand for?' differed significantly according to mode of response $\left(\chi^{2}(2)=8.114, P=0.017\right)$ with almost twice as many online respondents (51\%) correctly naming what all three letters stood for compared to paper respondents (27\%). Online respondents were also more likely to correctly indicate a causal 
relationship between HPV and cervical cancer $\left(\chi^{2}(1)=5.000, P=0.025 ; \mathrm{OR}=2.416,95 \% \mathrm{Cl}=\right.$ $1.105-5.283$ ) and to correctly answer that the HPV vaccine would not prevent all cases of cancer $\left(\chi^{2}(1)=9.168, P=0.002 ; \mathrm{OR}=3.236,95 \% \mathrm{Cl}=1.491-7.022\right)$. Although $73 \%$ of online respondents correctly described HPV as a virus, infection or STI/STD compared to $59 \%$ of paper respondents and $65 \%$ of online responsdents knew that HPV could be contracted through either sexual intercourse or sexual contact compared to $50 \%$ of paper respondents, these differences did not reach significance.

The number of missed or 'don't know' answers for HPV knowledge questions also differed according to mode of response $(t(125)=-2.358, P=0.020)$ with paper respondents missing a mean of $1.55(S D=1.413)$ questions compared to a mean of $0.99(S D=1.293)$ questions for online respondents. Paper respondents were more likely to give a blank or 'don't know' response for the question 'What is the relationship, if any, between HPV and cervical cancer?' $\left(\chi^{2}(1)=4.919, P=0.027 ; \mathrm{OR}=2.525,95 \% \mathrm{Cl}=1.099-5.798\right)$ and for the question 'Do you think the HPV vaccine will prevent all cases of cervical cancer?' $\left(\chi^{2}(1)=\right.$ 4.067, $P=0.044 ; \mathrm{OR}=2.520,95 \% \mathrm{Cl}=1.009-6.297)$.

Most women reported having heard about HPV from school (74\%) or from a health professional (62\%) with fewer women having heard about it from friends and family (35\%). These sources of information did not differ according to mode of response. However, online respondents were more likely to have heard of HPV from the internet $\left(\chi^{2}(1)=5.399, P=\right.$ $0.020 ; \mathrm{OR}=2.532,95 \% \mathrm{Cl}=1.144-5.604)$ or newspapers $\left(\chi^{2}(1)=6.400, P=0.011 ; \mathrm{OR}=\right.$ $4.732,95 \% \mathrm{Cl}=1.296-17.281)$ and there was a trend towards them being more likely to have heard about it from television $\left(\chi^{2}(1)=3.511, P=0.061 ; \mathrm{OR}=2.353,95 \% \mathrm{Cl}=0.947-\right.$ $5.844)$. 


\section{DISCUSSION}

Just over a quarter of women named HPV as a cause of cervical cancer in response to an open-ended question. This represents a considerable increase compared to previous studies of UK samples, prior to the introduction of the HPV vaccination programme (Waller et al, 2004; Marlow et al., 2007). The open-ended questions used in this study to elicit knowledge of the causes of cervical cancer were identical to those used in one of the previous studies which found that HPV was only mentioned by $2.5 \%$ of women (Marlow et al., 2007). This suggests that knowledge of the causal link between HPV and cervical cancer has improved substantially since the introduction of the HPV vaccination programme in 2008 , although see discussion point below about using university students.

In addition, the current study found that three quarters of women reported that they had heard of HPV. Again, this signifies a large increase in awareness of HPV since the HPV vaccination programme began compared to prior studies which found that approximately one third had heard of HPV (Pitts \& Clarke, 2002; Phillips et al., 2003; Waller et al., 2003; Marlow et al., 2007).

Furthermore, of those women who reported being aware of HPV, knowledge was reasonably good with $50 \%$ of women scoring at least 4 out of 5 on questions about HPV knowledge. The mean number of correct responses to the 5 HPV questions was 2.989. Compared to previous studies, which showed that women who had heard of HPV generally had a very poor knowledge of it (Pitts \& Clarke, 2002; Walsh et al,. 2008) this demonstrates a great improvement.

Lastly, and importantly, there were significant differences in performance between those participants who completed the paper questionnaire and those who complete the 
online version. Women who completed the online questionnaire were more likely to name HPV as a cause of cervical cancer, to name smoking and the contraceptive pill as causes, to have heard of HPV, to know what the letters HPV stand for and to know that the HPV vaccine does not prevent all cases of cancer. This is consistent with research which found online respondents to have better knowledge about cholesterol than face to face respondents (Duffy et al., 2005).

Three quarters of the women reported that they had received or were offered the HPV vaccination and it is likely that the observed increase in HPV knowledge relative to previous studies will have occurred due to the implementation of the UK vaccination programme.

Whilst this study seems to show a considerable improvement in awareness and knowledge of HPV and cervical cancer since the introduction of the UK vaccination programme, it is possible that in fact this is due to our use of university students. Previous studies have shown that awareness of cervical cancer and the associated risk factors increases with educational attainment (e.g., Low et al 2012; Waller et al, 2004). However, despite the relatively high educational status of our sample, there is clearly still a significant number of women who are lacking information and knowledge on the subject. Over three quarters of women did not name HPV as a cause of cervical cancer with a half not mentioning HPV or an unspecified STI/STD and a quarter of women had not heard of HPV. It was also observed that a considerable number of women had heard of HPV but did not name HPV, STI/STDs or any sexual factor as a cause of cervical cancer. This may suggest that whilst knowledge of HPV and its relationship with cervical cancer may be increasing, there are still a number of women who have an increased awareness of HPV but lack understanding as to what the implications of contracting HPV are. 
Even amongst those women who had heard of HPV, gaps in knowledge or misconceptions were still apparent. A third of women who had heard of HPV could not say what HPV is, almost a half did not know how it is contracted, and just under a third did not know that it can cause cervical cancer or that the HPV vaccine will not prevent all cases of cervical cancer. Clearly, this lack of knowledge could have implications for women's understanding of their own risk of cervical cancer and decisions to participate in HPV vaccination and cervical screening programmes. It is of particular concern given that all bar 5 of our sample were eligible for the HPV vaccination or catch up programme, suggesting that an opportunity to educate young women is being missed, although we acknowledge that further increases in awareness may occur once the majority of the cohort who are not currently eligible for screening receive their first invitation to screening.

Crucially, there were differences between the responses to the questions depending on whether participants received an online or paper questionnaire to complete. When Duffy et al (2005) observed the same difference, they considered the nature of their respondents further. They found that face to face respondents with home or work internet access performed better than face to face respondents without internet access and consluded that their online participants were not necessarily looking up the answers, but rather they were simply likely to be better informed as internet users than non-internet users. Although we cannot be certain that our findings are not also due to differences in the two groups of participants, the lack of difference in demographics between the two groups and the fact that they were all students (and therefore all have internet access) renders this interpretation unlikely. Another possibility is that a significant proportion of the online participants also researched their answers online whilst completing the questionnaire. This conclusion is hinted at by the fact that online participants were significantly more likely to 
name smoking, the contraceptive pill and genetic factors as causes of cervical cancer. These are all mentioned as possible causes on the Cancer Research UK website, which is the first website which comes up when 'causes of cervical cancer' is entered into the search engine Google. One participant even wrote in one of the open ended questions "I genuinely had no idea so just googled it". An additional piece of evidence supporting this conclusion is that $40 \%$ of participants in the online condition identified the internet as one of the sources they had heard of HPV from, significantly higher than the $21 \%$ of participants in the paper-based condition. The finding of differences in responses between the two groups, particularly given the apparently higher levels of knowledge and understanding in the online group, suggests that caution should be exercised when interpreting data from online studies. Lastly, the experimenters were in the room with the paper-based participants, making online searches less likely although not impossible. Findings of a significant increase in knowledge from such studies may well be inflating the true extent of that increase.

\section{Conclusion}

This study demonstrates that knowledge and awareness of HPV and its link to cervical cancer appears to have increased in female students since the implementation of the HPV vaccination programme. However, there is still a considerable number of women who have little to no knowledge of HPV. If women are to be able to make informed decisions about participating in HPV vaccination and cervical screening, improvements need to be made in the dissemination of information about HPV. Furthermore, care should be taken when interpreting data from online studies as it is likely that these inflate the extent of knowledge related responses. 
Declaration of Interest.

The authors report no conflicts of interest. 


\section{REFERENCES}

Duffy, B., Smith, K., Terhanian, G., \& Bremer, J. 2005. Comparing data from online and faceto-face surveys. International Journal of Market Research, 47: 615.

Low, E. L., Simon, A. E., Lyons, J., Romney-Alexander, D., \& Waller, J. (2012). What do British women know about cervical cancer symptoms and risk factors? European Journal of Cancer, 48: 3001-3008.

Marlow LAV, Waller J, Wardle J. 2007. Public awareness that HPV is a risk factor for cervical cancer. British Journal of Cancer, 97: 691-694

Marlow LAV, Zimet GD, McCaffery KJ, Ostini R, Waller J. 2013. Knowledge of human papillomavirus (HPV) and HPV vaccination: An international comparison. Vaccine 31: 763-769

Phillips Z, Johnson S, Avis M, Whynes DK. 2003. Human papillomavirus and the value of screening: Young women's knowledge of cervical cancer. Health Education Research, 18: $318-28$

Pitts M, Clarke T. 2002. Human papillomavirus infections and risks of cervical cancer: what do women know? Health Education Research, 17: 706-714

Waller J, McCaffery K, Wardle J. 2004. Beliefs about the risk factors for cervical cancer in a British population sample. Preventive Medicine, 38: 745-753

Waller J, McCaffery K, Forrest S, Szarewski A, Cadman L, Wardle J. 2003. Awareness of human papillomavirus among women attending a well women clinic. Sexually Transmitted Infections 79: 320-322 
Walsh CD, Gera A, Shah M, Sharma A, Powell JE, Wilson S. 2008. Public knowledge and attitudes towards Human Papilloma Virus (HPV) vaccination. BMC Public Health 8:

368

WHO/ICO Information Centre on HPV and Cancer (HPV Information Centre). 2013. Human papillomavirus and related diseases. Summary report. June 3, 2013. United Kingdom. http://www.hpvcentre.net/statistics/reports/GBR.pdf (accessed 10 August 2013)

${ }^{i}$ A copy of the questionnaire is available on request from the first author. 